\title{
Present status of Japanese ethics committees : a survey in Tokushima Prefecture
}

\author{
Rumi Katashima', Chiho Sato', Seizo Kinoshita², Shu Kawashima², \\ and Hiroaki Yanagawa \\ ${ }^{1}$ Clinical Trial Center for Developmental Therapeutics, Tokushima University Hospital, Tokushima, \\ Japan, ${ }^{2}$ Tokushima Medical Association, Tokushima, Japan
}

\begin{abstract}
Clinical research is important to improve medical quality, and ethics review is essential to conduct clinical research. Since the establishment of the first Japanese ethics committee at the University of Tokushima in 1982, Japanese ethics committees have increased. In this study, we surveyed the status of clinical studies and ethics committees in one Japanese region. The survey was conducted in collaboration with the Tokushima Medical Association. A questionnaire was established and mailed to all medical institutions $(n=737)$ registered to the Tokushima Medical Association in 2012. Among 737, 223 (30.3\%) questionnaires were returned and 221 were completed and are included in this analysis (respondents). Among respondents, 51 (23.1\%) had performed clinical research, and of these, 17 had established ethics committees (though one was omitted from the following analysis due to an unsatisfactory response). Among 16 ethics committees, review of protocol amendments, review of serious adverse events, annual follow - up of approved protocols, and education for committee members were active in $10(62.5 \%), 9(56.3 \%), 6(37.5 \%)$ and $4(25.0 \%)$, respectively. Research ethics education was active in 4 (25.0\%). Based on the results, we attempt to establish an appropriate system for ethical conduct of health-related research in Tokushima Prefecture. J. Med. Invest. 61 : 399-403, August, 2014
\end{abstract}

Keywords : ethics committee, research ethics, clinical research, regional area

\section{INTRODUCTION}

Ethics review by an independent committee is essential for research involving human subjects. In Japan, clinical trials leading to the approval of drugs (registration trials) are regulated by pharmaceutical affairs laws. In 1998, Good Clinical Practice (GCP), originally approved by the International Conference on Harmonisation of Technical Requirements for Registration of Pharmaceuticals for Human Use

Received for publication May 30, 2014 ; accepted July 7, 2014.

Address correspondence and reprint requests to Rumi Katashima, Ph.D., Clinical Trial Center for Developmental Therapeutics, Tokushima University Hospital, Kuramoto-cho, Tokushima, 770-8503 Japan and Fax : +81-88-633-9295.
(ICH) was effectively deployed in all registration trials, and registration trials are reviewed by committees that meet GCP. In contrast, engaging an ethics committee to review investigator-initiated research took place for the first time in Japan at the University of Tokushima in 1982, not as a response to national directives. Gradually, ethics committees in Japan have increased in number, and by the 1990s, Saito (1) surveyed 80 medical schools by questionnaire in 1991 and reported that ethics committees had been established in 79 of them. Akabayashi et al. reported the status of Japanese medical schools and the number of general hospital ethics committees in 1997 (2), as well as the number of ethics committees in medical organizations in 1998 (3).

Although originally Japanese ethics committees 
were established voluntarily, after the enforcement of ethical guidelines by the ministries of Japan in 2001, ethics committees were required to act in accordance with these ethical guidelines. At the same time, investigators of medical institutions, such as clinics, found it difficult to establish their own ethics committees. Thus, requesting ethical review from the ethics committee of other medical institutions was encouraged. The ethics committee of Tokushima University Hospital started to review applications from other institutions in 2003, and communication between medical institutions in the regional area has become more important. Although Sasaguri et al. (4) reported the survey results on the status of ethics committees in Fukuoka Prefecture in 2007, there is still little information known about this particular region.

We surveyed the performance of clinical trials and the status of ethics committees in the Shikoku region of Tokushima Prefecture, a rural area of Japan, and reported the survey results. Considering the situation in Japan as described above, in the present article, "ethics committee" is defined as a committee that reviews investigator-initiated research and is primarily established in a medical institution.

\section{METHODS}

The ethics committee of Tokushima University Hospital began reviewing applications from other institutions in 2003. At first, we analyzed the records of the ethics committee of Tokushima University Hospital to determine the number of applications from other medical institutions.

We assessed the performance of clinical trials and the status of ethics committees in Tokushima Prefecture by using a cross-sectional study in collaboration with the Tokushima Medical Association.

A questionnaire was specifically designed for use in this study. The questionnaire was anonymous and contained two parts with 24 questions. The first part consisted of two questions to determine the performance of clinical trials in each medical institution. The second part consisted of 22 questions, such as the presence of ethics committees, their activities, their structure, and education for the members. The questionnaire was provided to all medical institutions ( $\mathrm{n}=737$ ) registered to the Tokushima Medical Association in 2012. The consent of each institution was implied by filling out the questionnaire.

Due to the limited number of medical institutions included in this study, no statistical analysis of the data was performed.

This study was approved by the Ethics Committee of Tokushima University Hospital.

\section{RESULTS}

Applications from other medical institutions for ethics review by the ethics committee of Tokushima University Hospital

The ethics committee accepts applications for review of studies conducted in institutes other than the University of Tokushima when the studies are simultaneously conducted in the University of Tokushima. From April, 2003 to March, 2014, all 1780 protocols from investigators of the University of Tokushima were reviewed, and among these, 274 applications from other institutions were reviewed.

\section{Respondents and their clinical research performance status}

Among the 737 medical institutions registered to the Tokushima Medical Association, 223 (30.3\%) questionnaires were returned and 221 (30.0\%) were completed and included in this analysis (respondents). Table 1 reports the attributes of the respondents. The recovery of questionnaires in hospitals was significantly higher than clinics $(\mathrm{P}=0.0027)$. As shown in table 2, among respondents, 51 (23.1\%) had performed clinical research. The experience

Table 1. Attributes of subjects for this survey

\begin{tabular}{lccc}
\hline Medical institutions & $\begin{array}{c}\text { Respondents } \\
\text { N(\%) }\end{array}$ & $\begin{array}{c}\text { No response } \\
\text { N(\%) }\end{array}$ & $P$ value* \\
\hline Hospital $(\mathrm{n}=115)$ & $49(42.6)$ & $66(57.4)$ & 0.0027 \\
Clinic $(\mathrm{n}=622)$ & $174(28.0)$ & $448(72.0)$ & \\
\hline Total & $223(30.3)$ & $514(69.7)$ & \\
\hline
\end{tabular}

* Chi-square test

Table 2. Number of institutions with or without experience performing clinical research

\begin{tabular}{|c|c|c|c|}
\hline \multirow[t]{2}{*}{ Medical institutions } & \multicolumn{2}{|c|}{ Performing of clinical trials } & \multirow{2}{*}{$P$ value* } \\
\hline & $\begin{array}{c}\text { Eexperience } \\
\mathrm{N}(\%)\end{array}$ & $\begin{array}{c}\text { No experience } \\
\mathrm{N}(\%)\end{array}$ & \\
\hline Hospital $(n=49)$ & $21(42.9)$ & $28(57.1)$ & \multirow{2}{*}{0.0004} \\
\hline Clinic $(\mathrm{n}=172)$ & $30(17.4)$ & $142(83.0)$ & \\
\hline Total & $51(23.1)$ & $170(77.3)$ & \\
\hline
\end{tabular}

* Chi-square test 
rate of clinical trials in hospitals was significantly higher than clinics $(\mathrm{P}=0.0004)$. Answers from 170 medical institutions stated that the primary reasons for the lack of experience of conducting clinical research were no arrangement of an ethics committee, no interest, lack of available assistants, and no chance to participate (Figure 1). Regarding the willingness to participate, 50 medical institutions (29.4\%) responded that they were willing to conduct clinical research if the reasons stated above (no arrangement of an ethics committee, lack of available assistants, and no chance to participate) were overcome.

Status of ethics committees in respondent institutions

Among 51 medical institutions that had experience performing clinical research, 17 (33.3\%) answered to have established ethics committees, 31 (60.8\%) answered not to have established ethics committees, and $3(5.9 \%)$ provided no answer (Figure 2).

\section{Quality assurance of ethics committees}

We analyzed issues concerning quality assurance of ethics reviews in 17 medical institutions that established an ethics committee. Among 17, one was omitted from the following analysis due to an unsatisfactory answer. Among 16 ethics committees, $14(87.5 \%)$ were confident that all studies applied for ethical review. Review of protocol amendments and of serious adverse events were reviewed in 10 (62.5\%), and $9(56.3 \%)$ ethics committees, respectively. Annual follow-up of approved protocols was conducted in 6 committees (37.5\%) and disclosure of the review results was done in 6 committees (37.5\%) (Figure 3). As for the necessity to communicate among ethics committees to promote quality, 11 (68.8\%) agreed, 4 (25.0\%) were neutral and 1 (6.3\%) disagreed.

\section{Research ethics education for committee members and investigators}

Although 8 (50.0\%) realized the need for education of committee members, 4 (25.0\%) have opportunities for education. As for research ethics education for investigators, 10 (62.5\%) realized the need, and

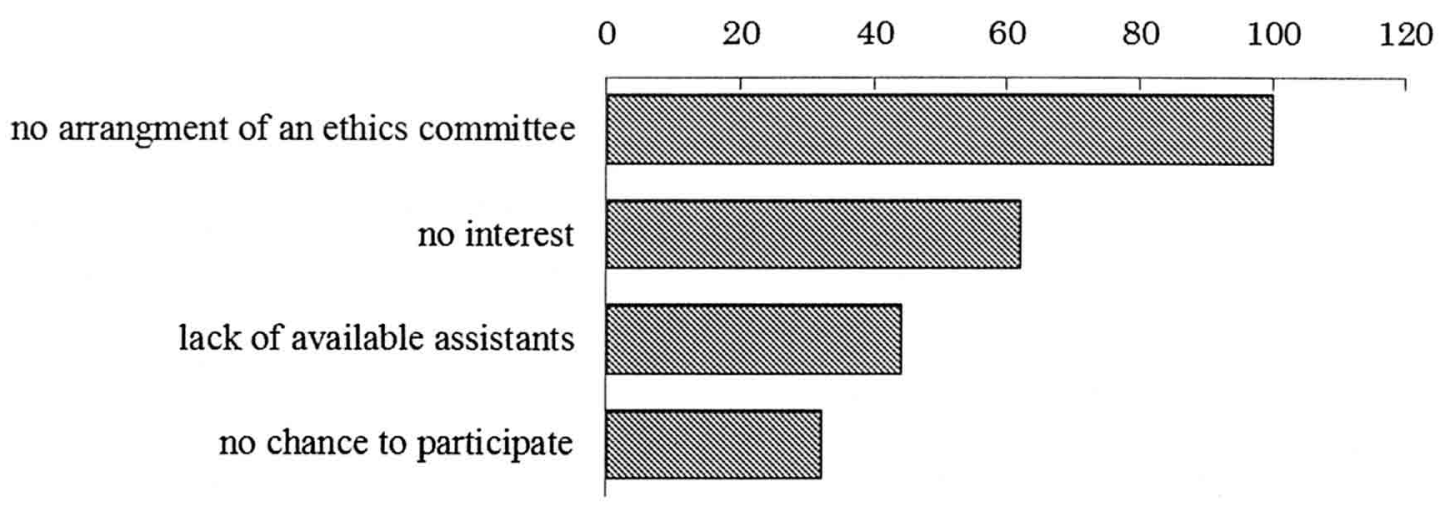

Figure 1 Primary reasons for the lack of experience of clinical research

Each respondent may give multiple reasons. Respondents intend to perform clinical research if their stated issues were resolved. The most common reason was that there was no arrangement for an ethics committee.

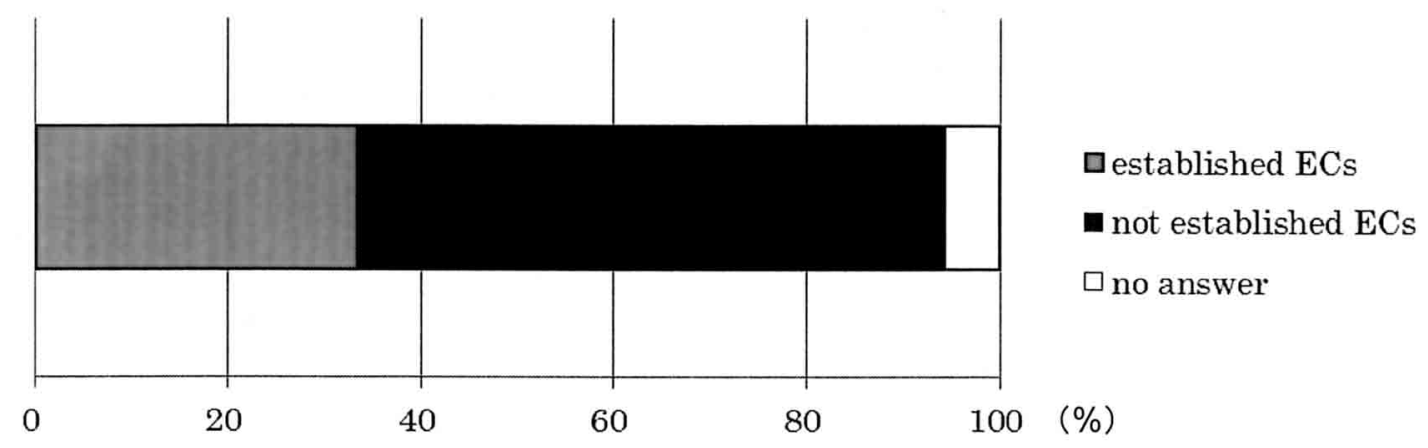

Figure 2 The status of ethics committees in 51 medical institutions

In 51 medical institutions with clinical research experience, 17 medical institutions (33.3\%) established an ethics committee (gray bar) ; 31 medical institutions (60.8\%) have not established an ethics committee (black bar) ; and 3 medical institutions (5.9\%) did not provide an answer (white bar). 


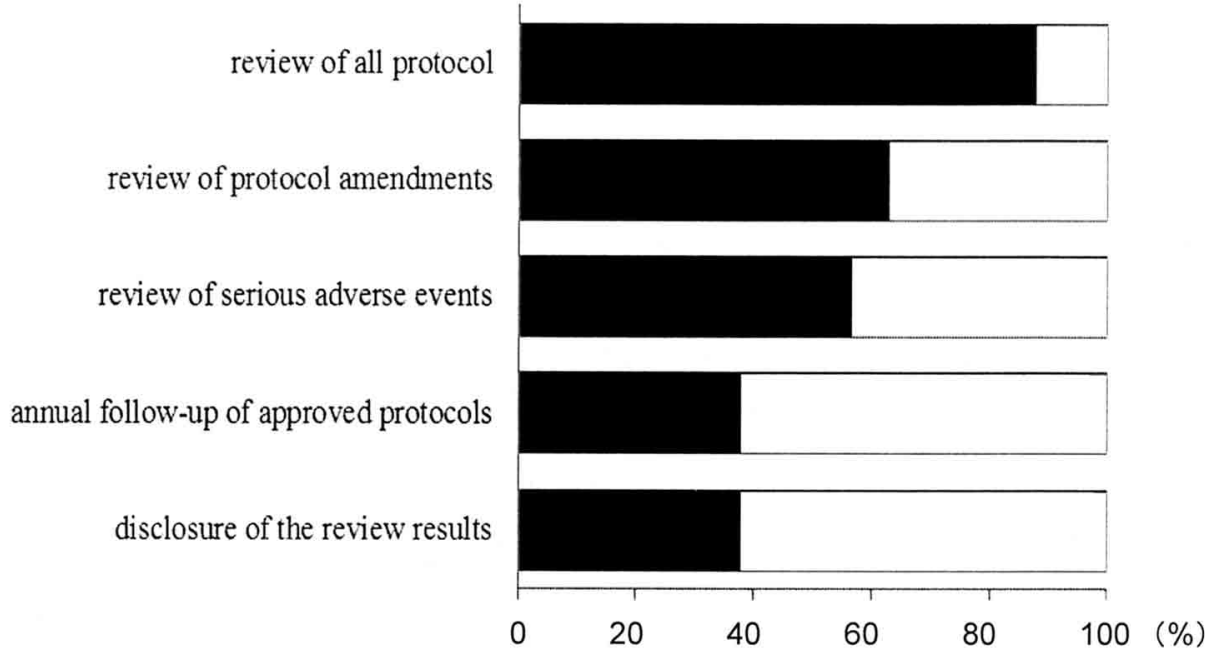

Figure 3 Quality assurance of ethics committees in the 16 medical institutions that have established ethics committees The figure shows the ratio of enforcement regarding the review of all protocols, review of protocol amendments, review of serious adverse events, annual follow-up of approved protocols, and disclosure of the review results in 16 medical institutions. The black bar shows that they have enforced the review. The white bar shows that they have not enforced the review.

$4(25.0 \%)$ answered they currently have opportunities for education. Regarding the desirable frequency for education opportunities, 3 medical institutions answered once every 6 months or once a year, and one answered 3 times every 6 months or once every 3 years.

\section{DISCUSSION}

To survey the status of ethics committees, the first issue is the selection of target institutions. In the survey in Fukuoka Prefecture (4), Sasaguri et al. selected hospitals and research institutions as targets, and omitted clinics from the survey. In the present study, we sent questionnaires to all medical institutions that were registered with the Tokushima Medical Institution, including clinics. We did this because this survey was planned around the Tokushima Medical Institution, and we wanted to know the complete status, including the performance of clinical research, in each medical institution. Sasaguri et al. (4) reported on 137 committees, and among these, 71 committees reviewed registration trials only. That means the remaining 66 were "ethics committees" as defined above in the present article. The recovery was rather low (30.3\%), and we found 17 ethics committees in Tokushima Prefecture. Considering the population of the two prefectures (Fukuoka with 5 million and Tokushima with 0.8 million), we obtained many answers from medical institutions, and considered them worth analyzing.
Originally, ethics committees in Japan were voluntarily established. After the enforcement of governmental guidelines, such as the Ethical Guideline for Human Genome and Gene Analysis Research in 2001, the Ethical Guideline for Epidemiological Research in 2002, and the Ethical Guideline for Clinical Studies in 2003, ethics committees became mandatory for medical institutions that conduct this type of research. The Governmental Ethical Guideline for Clinical Studies was revised and enforced in 2009. Under the revised guideline, administrators of the committee must open the activity to the public and report to the ministry annually. The provision for compensation for clinical studies on the evaluation of drugs and medical devices has been added. Nevertheless, less than half of the institutions ( $\mathrm{n}=$ $6,37.5 \%$ ) answered that they do annual follow-up of approved protocols. As for research ethics education for committee members and investigators, one quarter of institutions answered that they have opportunities for education, though more than half of the institutions realized the need.

An important issue for Japanese ethics committees is quality assurance. Although the structure and functions are basically defined in governmental ethics guidelines, a system to assure the quality of ethics committees is still unsatisfactory. The ethical guidelines for Epidemiological Research and Clinical Studies are to be integrated soon. In the new draft guidelines, a paragraph about quality assurance is being considered. Moreover, a governmental certification system for ethics committees is also being 
discussed. In this survey, it was suggested that a system with an acceptable level of oversight was currently in place to review all protocols before the research begins, but the system was not developed enough to review the amendment of protocols, serious adverse events, and annual follow-up of approved protocols. While in the ethics committee of each medical institution, it is difficult to observe the governmental ethical guidelines completely to assure the quality of the committees. Until now, each institution and hospital attempted to establish its own ethics committee. Now, the use of one ethics committee by several institutions is being discussed in Japan for quality assurance.

Tokushima University Hospital established the "Tokushima Network for Clinical Trials" (TNCT) in collaboration with the Tokushima Medical Association in 2004 (5). This step was originally planned for Tokushima University Hospital to contribute in the region by promoting registration trials. The committee of Tokushima University Hospital that reviews registration trials also reviews registration trials in medical institutions that are registered to the TNCT free of charge in the event the same registration trial was done at Tokushima University Hospital. Like local research ethics committees in the UK (6), it is an effective strategy to have the ethics committee of Tokushima University Hospital play a role as a local research ethics committee. Practical issues, such as financing, should be resolved to encourage this strategy.

In the present study, we investigated the status of ethics review for clinical research in one region of Japan. Based on the results, we will continue to support establishing an appropriate system for the ethical conduct of health-related research in Tokushima Prefecture.

\section{CONFLICT OF INTEREST}

The authors have no conflicts of interest to declare.

\section{ACKNOWLEDGEMENTS}

The authors would like to thank Prof. Noriaki Takeda, the chairman of the Ethics Committee of Tokushima University Hospital for his encouragement and support. We would also like to thank Ms. Michiko Yoshimaru, Reiko Tomioka, Kimiko Fukuchi, and Mitsuko Maruzasa for their assistance in data collection. We thank Ms. Toshiko Miyamoto and Makiko Yamagami for their helpful discussion and also the members of the Clinical Trial Center for Developmental Therapeutics, Tokushima University Hospital, for their kind support.

\section{REFERENCES}

1. Saito $\mathrm{T}$ : Ethics committees in Japanese medical schools. HEC Forum 4(4) : 281-7, 1992

2. Akabayashi A, Slingsby BT, Nagao N, Kai I, Sato $\mathrm{H}$ : An eight-year follow-up national study of medical school and general hospital ethics committees in Japan. BMC Med Ethics $8: 8$, 2007

3. Akabayashi A, Slingsby BT, Nagao N, Kai I, Sato $\mathrm{H}$ : A five year follow-up national study of ethics committees in medical organizations in Japan. HEC Forum 20(1) : 49-60, 2008

4. Sasaguri T, Shibata T, Ueguchi M, Shiraishi F, Miwa Y, Takahashi F, Morimoto S : Questionnaire survey of the institutional review boards in Fukuoka aiming at the construction of the board member education system. Clin Eval 36 : 393-419, 2008 (in Japanese, English Abstract)

5. Yanagawa H, Irahara M, Kawashima S, Kagawa S : Tokushima Network for Clinical Trials. The views of doctors on registration trials in a Japanese rural area : a survey of medical institutions registered to the Tokushima Network for Clinical Trials. J Int Med Res, 36(5) : 1117-1122, 2008

6. Kerrison S, Pollock AM : The reform of UK research ethics committees : throwing the baby out with the baby water? J Med Ethics, 31 : 487-489, 2005 